\title{
Three loop DIS and transversity operator anomalous dimensions in the RI' scheme
}

\author{
J.A. Gracey* \\ Theoretical Physics Division, Department of Mathematical Sciences, University of Liverpool, \\ P.O. Box 147, Liverpool, L69 3BX, United Kingdom. \\ E-mail: graceyeliv.ac.uk
}

We discuss the computation of the three loop anomalous dimensions for various operators used in deep inelastic scattering in the $\overline{\mathrm{MS}}$ and $\mathrm{RI}^{\prime}$ schemes. In particular the results for the $n=5$ and 6 Wilson operators in arbitrary linear covariant gauge in the $\mathrm{RI}^{\prime}$ scheme are new.

XI International Workshop on Advanced Computing and Analysis Techniques in Physics Research April 23-27 2007

Amsterdam, the Netherlands

${ }^{*}$ Speaker. 


\section{Introduction}

The use of symbolic manipulation and computer algebra has been an invaluable tool for providing large loop order results in quantum field theory in general and in quantum chromodynamics (QCD) in particular. The outstanding example is that of the full three loop anomalous dimensions for flavour non-singlet and singlet unpolarized operators for deep inelastic scattering as an analytic function of the operator moment $n,[1,2,3,4]$. Also the Wilson coefficients have been provided to the same precision. This large project, of the order of ten years, required not only the extensive use of the symbolic manipulation programme FORM, [5], but also its own development to handle the unforeseen complexity of the computation. An earlier approach, [6, 7], to this problem in deep inelastic scattering was to determine results for fixed (even) moments using the MINCER algorithm, [8], translated into FORM, [9]. For instance, the first few moments were given in [6, 7] and subsequently those for $n=12$ and $n=16$ appeared in $[10,11]$. At that time the fixed moment expressions were used to parametrize the full expressions but such an approach was clearly incomplete lacking the correctness of a full evaluation. However, the results subsequently served as very important independent checks on the final arbitrary $n$ results. Now that the computational algorithm has been established, in principle it can be applied to other operators underlying related phenomenology. For example, the case of polarized Wilson operators will be relevant for spin physics. In addition in the spin context there is interest in a similar operator called transversity, [12, 13, 14]. This corresponds to the probability of finding a quark in a transversely polarized nucleon polarized parallel to the nucleon versus that of the nucleon in the antiparallel polarization. From a theoretical point of view it is similar to the non-singlet unpolarized Wilson operator but experimentally it is not as accessible since there is no direct coupling to quarks. Nevertheless there have been proposals to study it at RHIC. Therefore, whilst in principle it is possible to calculate the arbitrary moment three loop transversity operator anomalous dimensions in the $\overline{\mathrm{MS}}$ scheme, it would be important to have strong independent checks on any future full result. Akin to the 1990's approach for the Wilson operator there is therefore a need for fixed moment calculations. Aside from this motivation, there is a secondary one.

One of the ingredients necessary to study the structure functions is the measurement of the non-perturbative matrix elements. From the theory point of view, a tool which achieves this is lattice regularization and various groups, such as QCDSF, have developed a substantial programme to determine key matrix elements. (See, for instance, $[15,16,17]$.) However, one technical aspect of such work is ensuring that the results agree in the continuum with expectations from the ultraviolet limit. One approach in this respect is for the lattice results to be matched onto the perturbative expressions in the chiral limit, where to aid precision one would prefer the results to as high a loop order as is calculationally feasible. This has been considered in a series of articles, $[18,19,20]$, to three loops. One technical issue is that to keep time (and money) to a minimum, the lattice computations are performed in renormalization schemes known as regularization invariant (RI) and its modification, $\mathrm{RI}^{\prime}$, [21, 22]. Unlike the $\overline{\mathrm{MS}}$ scheme, they are mass dependent renormalization schemes. Results in this scheme have then to be converted to the standard $\overline{\mathrm{MS}}$ scheme. In the continuum QCD has been renormalized at high loop order in both RI and RI' in $[23,18]$ and the conversion functions established for various quantities of interest. Therefore to aid lattice computations of the matrix elements one requires the finite part of the analogous Green's functions but 
only for low moment since clear signals for higher moments information are hard to extract from the numerical noise on the lattice. Given the need for such accurate results for a specific Green's function on the lattice, not only for the transversity operators but also for the Wilson operators, we report the results of recent computations in this area, [20, 24]. One consequence of the Green's function considered is that the operator anomalous dimensions emerge as a corollary in the RI' and $\overline{\mathrm{MS}}$ schemes. In addition to the results already available, [18, 19, 20, 24], we will provide the RI' anomalous dimensions for the $n=5$ and 6 Wilson operators in arbitrary covariant gauge. Although the finite parts are required for lattice calculations for low moment, our computations have been extended to high moment for the transversity operator but only to determine the anomalous dimensions. At the appropriate point we will mention some of the computer algebra aspects of this and previous work which without the power of FORM would have rendered the determination of any result virtually impossible.

The paper is organised as follows. In section two we introduce our notation and computational strategy in more detail before discussing the appropriate points of the RI' scheme in section three. Section four details a simple low moment example, whilst the symbolic manipulation issues are recorded in the context of the higher moment calculation in section five. The explicit anomalous dimensions are given in section six with a few concluding remarks provided in the final section.

\section{Background}

The two basic classes of operators we will consider are the non-singlet Wilson operators

$$
\mathscr{O}_{W}^{v_{1} \ldots v_{n}}=\mathscr{S} \bar{\psi}^{i} \gamma^{v_{1}} D^{v_{2}} \ldots D^{v_{n}} \psi^{j}
$$

and the transversity operators

$$
\mathscr{O}_{T}^{\mu v_{1} \ldots v_{n}}=\mathscr{S} \bar{\psi}^{i} \sigma^{\mu v_{1}} D^{v_{2}} \ldots D^{v_{n}} \psi^{j}
$$

where $\psi^{i}$ is the quark field, $1 \leq i \leq N_{f}$ for $N_{f}$ quark flavours, $D_{\mu}$ is the covariant derivative and $\sigma^{\mu v}=\frac{1}{2}\left[\gamma^{\mu}, \gamma^{v}\right]$. The operation of totally symmetrizing with respect to the Lorentz indices $\left\{v_{i}\right\}$ and ensuring the operator is traceless is denoted by $\mathscr{S}$ where the respective but different tracelessness conditions are given by

$$
\eta_{v_{i} v_{j}} \mathscr{O}_{W}^{v_{1} \ldots v_{i} \ldots v_{j} \ldots v_{n}}=0
$$

and, [25],

$$
\eta_{\mu v_{i}} \mathscr{O}_{T}^{\mu v_{1} \ldots v_{i} \ldots v_{n}}=0 \quad(i \geq 2) \quad, \quad \eta_{v_{i} v_{j}} \mathscr{O}_{T}^{\mu v_{1} \ldots v_{i} \ldots v_{j} \ldots v_{n}}=0
$$

For (2.2) the anomalous dimensions are available for all moments $n$ at one and two loops in the $\overline{\mathrm{MS}}$ scheme in $[26,27,28,25,29]$. At three loops fixed moment results are available for moments up to $n=8$ for $\overline{\mathrm{MS}}$ and $n=7$ for $\mathrm{RI}^{\prime}$, [19, 20, 24]. The specific Green's functions relevant for the lattice matching are

$$
\begin{aligned}
G_{W}^{v_{1} \ldots v_{n}}(p) & =\left\langle\psi(-p) \mathscr{O}_{W}^{v_{1} \ldots v_{n}}(0) \bar{\psi}(p)\right\rangle \\
G_{T}^{\mu v_{1} \ldots v_{n}}(p) & =\left\langle\psi(-p) \mathscr{O}_{T}^{\mu v_{1} \ldots v_{n}}(0) \bar{\psi}(p)\right\rangle
\end{aligned}
$$


where the operator is inserted at zero momentum. This allows for the application of the MINCER algorithm, $[8,9]$, which determines the finite part of scalar massless two point functions using dimensional regularization in $d=4-2 \varepsilon$ dimensions to three loops. Unlike earlier approaches to extract anomalous dimensions we do not contract the free Lorentz indices with a null vector. Whilst such a contraction has the effect of excluding the trace terms in the operator itself or the Green's function decomposition, the main reason why we cannot follow that route here is that the lattice makes measurements in different directions of the momentum components. This allows for the extraction of the values of each of the individual amplitudes into which the Green's functions are decomposed.

From the form of the operators there will be $n n$-point Feynman rules for both the Wilson and transversity operators, each with two quark legs. However, at the three loop order we will work at, only the Feynman rules up to and including three gluon legs will be necessary. Hence, there are 3 one loop, 37 two loop and 684 three loop Feynman diagrams contributing to each Green's function. These are generated electronically using the QGRAF package, [30], before being converted into FORM input notation to allow for the application of the FORM version of the MINCER package, $[8,9]$. As the MINCER algorithm is only applicable to scalar Feynman integrals, for each moment $n$ the Green's functions, (2.5), need to be decomposed into invariant amplitudes and Lorentz tensors which respect all symmetry structures. Whilst we will discuss more details later, it suffices to note at this point that for Wilson operators there will be two independent amplitudes but three for the transversity case.

Although the lattice computations are ultimately performed in the Landau gauge, we will compute in an arbitrary linear covariant gauge. The associated gauge parameter will act as an internal checking parameter since, for instance, in the $\overline{\mathrm{MS}}$ scheme the anomalous dimension of gauge invariant operators are independent of the gauge parameter, [31, 32]. As the computations are clearly automatic we employ the procedure of [33] where all the diagrams are computed for bare coupling, $g_{0}$, and gauge parameter $\alpha_{0}$. To extract the anomalous dimension the renormalization constant is fixed (either in the $\overline{\mathrm{MS}}$ or $\mathrm{RI}^{\prime}$ schemes) by rescaling these variables by the known coupling constant and gauge parameter renormalization constants

$$
g_{\mathrm{o}}=Z_{g} g \quad, \quad \alpha_{\mathrm{o}}=Z_{\alpha}^{-1} Z_{A} \alpha
$$

in our conventions, where $Z_{A}$ is the gluon wave function renormalization constant. The remaining divergence for each of the Green's function is absorbed into the operator renormalization constant together with a specified finite part in the case of the RI' scheme to leave the finite parts for the lattice matching. In practice the results are determined in the $\overline{\mathrm{MS}}$ scheme first, primarily due to more consistency checks being available before extracting the RI' expressions.

\section{3. $\mathbf{R I}^{\prime}$ scheme}

We briefly review the parts of the RI' scheme needed for the computations discussed here. Originally the scheme was invented in the context of the lattice, [21, 22], but it is not restricted to a discrete spacetime. The continuum analogue has been studied to three and four loop order in $[23,18]$. In general terms it is a mass dependent renormalization scheme where the renormalization of the quark field is chosen to be non-minimal in a way which is appropriate for lattice analyses. 
The coupling constant (and thus vertex) renormalization is performed in an $\overline{\mathrm{MS}}$ way and so in some sense the RI set of schemes sits between $\overline{\mathrm{MS}}$ and MOM type schemes. To reduce time (and cost), since taking a derivative on the lattice requires significant computation, the quark 2-point function, $\Sigma_{\psi}(p)$, is renormalized according to the $\mathrm{RI}^{\prime}$ prescription, [21, 22],

$$
\left.\lim _{\varepsilon \rightarrow 0}\left[Z_{\psi}^{\prime} \Sigma_{\psi}(p)\right]\right|_{p^{2}=\mu^{2}}=\not p
$$

where $\mu^{2}$ is the renormalization point. In other words there are no $O\left(g^{2}\right)$ corrections to $\Sigma_{\psi}(p)$ after renormalization at $p^{2}=\mu^{2}$ as these finite parts are absorbed into the quark wave function renormalization constant, $Z_{\psi}^{\prime}$. We use the notation throughout that a ' on a quantity indicates that renormalization has been performed in the $\mathrm{RI}^{\prime}$ scheme. Otherwise the scheme is $\overline{\mathrm{MS}}$. For completeness the RI scheme, which is not of interest to us here, involves taking a momentum derivative of $\Sigma_{\psi}(p)$ first before choosing the result to be the tree value at the renormalization point, $[21,22]$. As an extension of the RI' scheme in the continuum, the gluon and Faddeev-Popov 2point functions are renormalized analogous to (3.1). However, as most interest in general is in quark 2-point Green's functions, there is no real need to pursue this route, unless one was perhaps intending to consider supersymmetric theories. Similar to the lattice we are ultimately interested in converting results from $\mathrm{RI}^{\prime}$ to $\overline{\mathrm{MS}}$ and therefore the variables in each scheme need to be related. Using the standard conversion definitions

$$
\alpha^{\prime}=\frac{Z_{A}}{Z_{A}^{\prime}} \alpha, \quad a^{\prime}=\left(\frac{Z_{g}}{Z_{g}^{\prime}}\right)^{2} a
$$

where $a=g^{2} /\left(16 \pi^{2}\right)$, we have the one loop relations, [23, 18],

$$
\begin{aligned}
a^{\prime} & =a+O\left(a^{5}\right) \\
\alpha^{\prime} & =\left[1+\left(\left(-9 \alpha^{2}-18 \alpha-97\right) C_{A}+80 T_{F} N_{f}\right) \frac{a}{36}\right] \alpha+O\left(a^{2}\right) .
\end{aligned}
$$

The explicit expressions to three loops are available in [18]. Though it is worth noting that the Landau gauge is preserved in changing between $\mathrm{RI}^{\prime}$ and $\overline{\mathrm{MS}}$. To illustrate the effect the schemes have on the basic anomalous dimensions, we note

$$
\begin{aligned}
& \gamma_{\psi}(a)=\alpha C_{F} a+\frac{1}{4}\left[\left(\alpha^{2}+8 \alpha+25\right) C_{A} C_{F}-6 C_{F}^{2}-8 C_{F} T_{F} N_{f}\right] a^{2}+O\left(a^{3}\right) \\
& \gamma_{\psi}^{\prime}(a)=\alpha C_{F} a+\left[\left(9 \alpha^{3}+45 \alpha^{2}+223 \alpha+225\right) C_{A}-54 C_{F}-(80 \alpha+72) T_{F} N_{f}\right] \frac{C_{F} a^{2}}{36}+O\left(a^{3}\right)
\end{aligned}
$$

where the group theoretic quantities are defined by

$$
\operatorname{Tr}\left(T^{a} T^{b}\right)=T_{F} \delta^{a b}, T^{a} T^{a}=C_{F} I, \quad f^{a c d} f^{b c d}=C_{A} \delta^{a b}
$$

for a colour group with generators $T^{a}$ and structure functions $f^{a b c}$. Clearly the difference in the numerical structure in (3.5) only appears at two and higher loops.

For the flavour non-singlet operators we are interested in here, we follow a similar route to (3.1) for defining the operator renormalization constant in the $\mathrm{RI}^{\prime}$ scheme. Writing $\Sigma_{\mathscr{O}}^{(T)}(p)$ as the 
amplitude in the Lorentz decomposition of (2.5) which contains the tree, $(T)$, part of the operator, we set

$$
\left.\lim _{\varepsilon \rightarrow 0}\left[Z_{\psi}^{\prime} Z_{\mathscr{O}}^{\prime} \Sigma_{\mathscr{O}}^{(T)}(p)\right]\right|_{p^{2}=\mu^{2}}=\mathscr{T}
$$

where $\mathscr{T}$ is the value of the tree term of amplitude, which may not necessarily be unity given the specific (non-unique) way of carrying out the decomposition.

\section{Simple example}

We now illustrate the preceeding remarks by discussing the case of the $n=2$ transversity operator in more detail, [19]. First, given the symmetry properties (2.4) the explicit traceless symmetrized operator is

$$
\begin{aligned}
\mathscr{S} \bar{\psi} \sigma^{\mu v} D^{\rho} \psi= & \bar{\psi} \sigma^{\mu v} D^{\rho} \psi+\bar{\psi} \sigma^{\mu \rho} D^{v} \psi-\frac{2}{(d-1)} \eta^{v \rho} \bar{\psi} \sigma^{\mu \lambda} D_{\lambda} \psi \\
& +\frac{1}{(d-1)}\left(\eta^{\mu v} \bar{\psi} \sigma^{\rho \lambda} D_{\lambda} \psi+\eta^{\mu \rho} \bar{\psi} \sigma^{v \lambda} D_{\lambda} \psi\right)
\end{aligned}
$$

in $d$-dimensions. Inserting (4.1) into the Green's function $G_{T}^{\mu v \rho}(p)$, it is decomposed into the three invariant amplitudes as

$$
\begin{aligned}
G_{T}^{\mu v \rho}(p)= & \Sigma_{T}^{(1)}(p)\left(\sigma^{\mu v} p^{\rho}+\sigma^{\mu \rho} p^{v}-\frac{(d+2)}{p^{2}} \sigma^{\mu \lambda} p^{v} p^{\rho} p_{\lambda}+\eta^{v \rho} \sigma^{\mu \lambda} p_{\lambda}\right) \\
& +\Sigma_{T}^{(2)}(p)\left(\eta^{\mu v} \sigma^{\rho \lambda} p_{\lambda}+\eta^{\mu \rho} \sigma^{v \lambda} p_{\lambda}-(d+1) \eta^{v \rho} \sigma^{\mu \lambda} p_{\lambda}\right. \\
& \left.\quad+\frac{(d-1)(d+2)}{p^{2}} \sigma^{\mu \lambda} p^{v} p^{\rho} p_{\lambda}\right) \\
& +\Sigma_{T}^{(3)}(p)\left(\sigma^{v \lambda} p^{\mu} p^{\rho} p_{\lambda}+\sigma^{\rho \lambda} p^{\mu} p^{v} p_{\lambda}+d \sigma^{\mu \lambda} p^{v} p^{\rho} p_{\lambda}-\eta^{v \rho} \sigma^{\mu \lambda} p_{\lambda} p^{2}\right)
\end{aligned}
$$

in $d$-dimensions. It is worth noting that this and other decompositions are not unique since one can always take a linear combination of the three (independent) tensor structures consistent with the symmetry and traceless properties to form another set of independent amplitudes. However, with this choice one can algebraically form a scalar object which is computed via MINCER. For instance, [19],

$$
\begin{aligned}
\Sigma_{T}^{(1)}(p)=-\frac{1}{8(d-1)(d-2)} \operatorname{tr}[ & \left(\sigma_{\mu \nu} p_{\rho}+\sigma_{\mu \rho} p_{v}-\frac{(d+2)}{p^{2}} \sigma_{\mu \lambda} p_{v} p_{\rho} p^{\lambda}\right. \\
& \left.\left.+\eta_{v \rho} \sigma_{\mu \lambda} p^{\lambda}\right) G_{T}^{\mu \nu \rho}(p)\right] \\
-\frac{1}{8(d-1)(d-2) p^{2}} \operatorname{tr}[ & \left(\sigma_{v \lambda} p_{\mu} p_{\rho} p^{\lambda}+\sigma_{\rho \lambda} p_{\mu} p_{v} p^{\lambda}\right. \\
& \left.\left.+d \sigma_{\mu \lambda} p_{v} p_{\rho} p^{\lambda}-\eta_{v \rho} \sigma_{\mu \lambda} p^{\lambda} p^{2}\right) G_{T}^{\mu v \rho}(p)\right] .
\end{aligned}
$$

This together with $\Sigma_{T}^{(i)}(p), i=2$ and 3, are the objects of interest for the lattice matching and have been determined to $O\left(a^{3}\right)$, [19]. For this specific example, we note that the construction of the tensor basis as well as the amplitude decomposition can easily be carried out by hand. This is 
primarily due to the small number of free Lorentz indices present. Clearly for the extraction of the anomalous dimensions and amplitudes for the higher moment operators, such a procedure would be unacceptably time consuming by hand. Moreover, it would be prone to elementary algebraic errors.

\section{Higher moment issues}

To extract the anomalous dimensions for the higher moment operators, it is clear that one has to proceed with a computer algebra construction to determine the basis for the independent amplitudes and hence the projections. We discuss the issues in relation to the $n=8$ transversity operator as an example, [24]. For this case there are initially seventeen potential tensors into which the Green's function (2.5) can be decomposed. These are built from the relevant vectors and tensors of the operator in question, which for transversity are $p^{\mu}, \eta^{\mu v}$ and $\sigma^{\mu v}$. The only constraint being that the Green's function has nine independent indices. Given these seventeen tensors then within FORM it is straightforward to construct the seventeen tensors which have the correct symmetry, but not traceless, properties. Taking a linear combination of these new objects with as yet unrelated coefficients, the relationship between these are fixed by imposing the remaining traceless criterion. In practical terms we take successive pairs of free indices and contract them. The coefficients of the resulting tensors produce constraints on the seventeen initial coefficients which can be solved. Whilst there are more contractions than coefficients there is redundancy in the system of linear equations which determine the coefficients. This is due to the symmetry of the operator itself. However, there is no unique solution and three coefficients remain unrelated producing three independent amplitudes. (For the Wilson operator the corresponding number is two.) For the higher moments, as we are ultimately interested in the anomalous dimensions, the specific linear combination one uses is not a major issue. The only constraint is to choose that projector of the three which leads to the lowest computation time when MINCER is applied. The test for this is to compare the run times for each projector to do the full two loop calculation in an arbitrary linear covariant gauge before generating the results for the three loop diagrams.

The other main computer algebra issue is the construction of the Feynman rules for each operator. Given that the Green's function has free indices one in principle has to construct the full symmetrized and traceless operator before applying the FORM routine to generate the explicit Feynman rules for the operator. However, given that the Green's function will be multiplied by a projector which is traceless, that part of the operator containing $\eta_{\mu \nu}$ tensors will automatically give zero upon contracting with the projector. Therefore there is no need to have an operator which is traceless; only an operator which is symmetrized will suffice. This will reduce computation time since otherwise with a traceless operator there will be an internal intermediate expression swell which will be sorted by FORM to produce the equivalent scalar expression as ignoring the traceless part. For instance, for the $n=8$ transversity operator the expression swell would have been substantial. Finally, in relation to the Feynman rules, only the part of the operator up to and including two quark and three gluon leg insertions are required for the full three loop computation. To illustrate the size of our higher moment calculation the FORM module containing the operator Feynman rule was 36 Mbytes for $n=7$ transversity and 300 Mbytes for $n=8$ transversity, [24]. Indeed the latter calculation could only be performed in the Feynman gauge rather than the full 
linear covariant or Landau gauges. Even then it took of the order of 40 days on a dual opteron 64 bit SMP 2 GHz machine. Hence, only the $\overline{\mathrm{MS}}$ result was determined with the RI' scheme anomalous dimension yet to be established.

As with all large computations carried out symbolically, it is worth detailing the various checks we used in order to be confident that our results are credible. First, for the case of the Wilson operators the three loop $\overline{\mathrm{MS}}$ anomalous dimensions are known, [1, 2, 3, 4], and our anomalous dimensions must therefore agree before extracting any finite parts for lattice matching. Moreover, for both Wilson and transversity operators the $\overline{\mathrm{MS}}$ expressions have been shown to be independent of the linear covariant gauge fixing parameter. For the transversity case we have the checks that the two loop anomalous dimensions must agree with $[26,27,28,25,29]$ for the various $n$ we consider. At three loops the only substantial check is that the residues of the poles in $1 / \varepsilon^{2}$ and $1 / \varepsilon^{3}$ have to agree with the renormalization group consistency check. In other words these are predicted from the one and two loop parts of the anomalous dimensions. In addition for the RI' scheme, one can compute the anomalous dimensions either directly from the renormalization constants deduced from the Green's function itself, or from the conversion functions, $C_{\mathscr{O}}(a, \alpha)$, based on the renormalization group. This is defined by

$$
C_{\mathscr{O}}(a, \alpha)=\frac{Z_{\mathscr{O}}^{\prime}}{Z_{\mathscr{O}}}
$$

where the renormalization constants are both expressed in terms of the variables defined in the same scheme. Then the RI' anomalous dimension is given by

$$
\gamma_{\mathscr{O}}^{\prime}\left(a^{\prime}\right)=\gamma_{\mathscr{O}}(a)-\beta(a) \frac{\partial}{\partial a} \ln C_{\mathscr{O}}(a, \alpha)-\alpha \gamma_{\alpha}(a) \frac{\partial}{\partial \alpha} \ln C_{\mathscr{O}}(a, \alpha) .
$$

(See, for example, [34].) Therefore, the expression on the left side will agree with the direct renormalization. For all the results presented in the next section we note that they all pass the checks discussed here.

\section{Results}

First, we record the explicit values for the anomalous dimensions of the Wilson operators $n=5$ and 6 in $\mathrm{RI}^{\prime}$ for arbitrary $\alpha$, which are new. The notation is that the numerical subscript denotes the moment whilst the superscript, $W$ or $T$, corresponds to either the Wilson or transversity operator respectively. We find

$$
\begin{aligned}
\gamma_{5}^{\prime W}(a)=\frac{91}{15} C_{F} a+\left[\left(33525 \alpha^{2}+100575 \alpha+1729270\right) C_{A}-156114 C_{F}-673880 T_{F} N_{f}\right] \frac{C_{F} a^{2}}{27000} \\
+\left[\left(30172500 \alpha^{4}+289359000 \alpha^{3}-97200000 \zeta_{3} \alpha^{2}+1409428125 \alpha^{2}\right.\right. \\
\left.-1004400000 \zeta_{3} \alpha+4758071625 \alpha-5142528000 \zeta_{3}+52067172425\right) C_{A}^{2} \\
+\left(23726250 \alpha^{3}+30956400 \alpha^{2}-415630950 \alpha\right. \\
\left.\quad+102384000 \zeta_{3}-9145680720\right) C_{A} C_{F}+6023484800 T_{F}^{2} N_{f}^{2} \\
-\left(268200000 \alpha^{2}-259200000 \zeta_{3} \alpha+1582173000 \alpha\right. \\
\left.+2514240000 \zeta_{3}+36792205400\right) C_{A} T_{F} N_{f}
\end{aligned}
$$




$$
\begin{aligned}
& +\left(107259600 \alpha+3680640000 \zeta_{3}-3053173120\right) C_{F} T_{F} N_{f} \\
& \left.+\left(1832544000 \zeta_{3}-829297168\right) C_{F}^{2}\right] \frac{C_{F} a^{3}}{48600000}+O\left(a^{4}\right)
\end{aligned}
$$

and

$$
\begin{aligned}
& \gamma_{6}^{\prime W}(a)=\frac{709}{105} C_{F} a+\left[\left(12116475 \alpha^{2}+36349425 \alpha+670295290\right) C_{A}\right. \\
&\left.-57119598 C_{F}-263443880 T_{F} N_{f}\right] \frac{C_{F} a^{2}}{9261000} \\
&+\left[\left(534336547500 \alpha^{4}+5228103069000 \alpha^{3}-1750329000000 \zeta_{3} \alpha^{2}\right.\right. \\
&+\quad 25439835416625 \alpha^{2}-18086733000000 \zeta_{3} \alpha+86004002776125 \alpha \\
&\left.-92121315552000 \zeta_{3}+988839358918775\right) C_{A}^{2} \\
&+\quad\left(355203339750 \alpha^{3}+158333464800 \alpha^{2}-9375191062650 \alpha\right. \\
&\left.\quad-5509035504000 \zeta_{3}-172078530172080\right) C_{A} C_{F} \\
&-\left(4749658200000 \alpha^{2}-4667544000000 \zeta_{3} \alpha+28468726629000 \alpha\right. \\
&+\left.+49102562880000 \zeta_{3}+704961641573000\right) C_{A} T_{F} N_{f} \\
&+\left(2419404145200 \alpha+73311557760000 \zeta_{3}-59288998908160\right) C_{F} T_{F} N_{f} \\
&+\left(31055615136000 \zeta_{3}-13674447985168\right) C_{F}^{2} \\
&+\left.117065906115200 T_{F}^{2} N_{f}^{2}\right] \frac{C_{F} a^{3}}{816820200000}+O\left(a^{4}\right)
\end{aligned}
$$

where $\zeta_{n}$ is the Riemann zeta function. As the Landau gauge is of particular interest, we record that the previous two expressions when $\alpha=0$ are

$$
\begin{aligned}
&\left.\gamma_{5}^{\prime W}(a)\right|_{\alpha=0}=\frac{91 C_{F}}{15} a+\left[864635 C_{A}-78057 C_{F}-336940 T_{F} N_{f}\right] \frac{C_{F} a^{2}}{13500} \\
&+ {\left[\left(52067172425-5142528000 \zeta_{3}\right) C_{A}^{2}+\left(102384000 \zeta_{3}-9145680720\right) C_{A} C_{F}\right.} \\
& \quad-\left(2514240000 \zeta_{3}+36792205400\right) C_{A} T_{F} N_{f}+6023484800 T_{F}^{2} N_{f}^{2} \\
&+\left(1832544000 \zeta_{3}-829297168\right) C_{F}^{2} \\
&\left.+\left(3680640000 \zeta_{3}-3053173120\right) C_{F} T_{F} N_{f}\right] \frac{C_{F} a^{3}}{48600000}+O\left(a^{4}\right)
\end{aligned}
$$

and

$$
\begin{aligned}
&\left.\gamma_{6}^{\prime W}(a)\right|_{\alpha=0}=\frac{709}{105} a+\left[335147645 C_{A}-28559799 C_{F}-131721940 T_{F} N_{f}\right] \frac{C_{F} a^{2}}{4630500} \\
&+\left[\left(988839358918775-92121315552000 \zeta_{3}\right) C_{A}^{2}\right. \\
& \quad-\left(5509035504000 \zeta_{3}-172078530172080\right) C_{A} C_{F} \\
&-\left(49102562880000 \zeta_{3}+704961641573000\right) C_{A} T_{F} N_{f} \\
&+\left(31055615136000 \zeta_{3}-13674447985168\right) C_{F}^{2} \\
&+\left(73311557760000 \zeta_{3}-59288998908160\right) C_{F} T_{F} N_{f} \\
&+\left.117065906115200 T_{F}^{2} N_{f}^{2}\right] \frac{C_{F} a^{3}}{816820200000}+O\left(a^{4}\right) .
\end{aligned}
$$


For further comparison between the schemes the $\overline{\mathrm{MS}}$ and RI' expressions for $n=5$ transversity are, [20],

$$
\begin{aligned}
\gamma_{5}^{T}(a)=\frac{92}{15} & C_{F} a+\left[189515 C_{A}-41674 C_{F}-79810 T_{F} N_{f}\right] \frac{C_{F} a^{2}}{6750} \\
+ & {\left[\left(190836000 \zeta_{3}+1975309075\right) C_{A}^{2}-\left(572508000 \zeta_{3}+325464235\right) C_{A} C_{F}\right.} \\
& \quad-\left(1192320000 \zeta_{3}+511395100\right) C_{A} T_{F} N_{f}+\left(381672000 \zeta_{3}-254723696\right) C_{F}^{2} \\
& \left.+\left(1192320000 \zeta_{3}-989903260\right) C_{F} T_{F} N_{f}-83718800 T_{F}^{2} N_{f}^{2}\right] \frac{C_{F} a^{3}}{12150000} \\
+ & O\left(a^{4}\right)
\end{aligned}
$$

and

$$
\begin{aligned}
\gamma_{5}^{T}(a)=\frac{92}{15} C_{F} a+\left[\left(30825 \alpha^{2}+92475 \alpha+1740690\right) C_{A}-166696 C_{F}-676560 T_{F} N_{f}\right] \frac{C_{F} a^{2}}{27000} \\
+\left[\left(194197500 \alpha^{4}+1854279000 \alpha^{3}-583200000 \zeta_{3} \alpha^{2}+8993896875 \alpha^{2}\right.\right. \\
\quad-6026400000 \zeta_{3} \alpha+30074295375 \alpha-37353312000 \zeta_{3} \\
+\quad 356401468700) C_{A}^{2}+\left(91239750 \alpha^{3}-209956950 \alpha^{2}\right. \\
\left.\quad-4997987400 \alpha+1076976000 \zeta_{3}-60979980560\right) C_{A} C_{F} \\
-\quad\left(1726200000 \alpha^{2}-1555200000 \zeta_{3} \alpha+10041363000 \alpha\right. \\
\left.\quad+17858880000 \zeta_{3}+253330505600\right) C_{A} T_{F} N_{f}+41629683200 T_{F}^{2} N_{f}^{2} \\
+\quad\left(1289803200 \alpha+27164160000 \zeta_{3}-22363266560\right) C_{F} T_{F} N_{f} \\
\left.+\left(10686816000 \zeta_{3}-7132263488\right) C_{F}^{2}\right] \frac{C_{F} a^{3}}{340200000}+O\left(a^{4}\right) .
\end{aligned}
$$

For completeness we record the next $\overline{\mathrm{MS}}$ anomalous dimensions in the sequence are, [20],

$$
\begin{aligned}
\gamma_{6}^{T}(a)= & \frac{34}{5} C_{F} a+\left[204770 C_{A}-42129 C_{F}-88810 T_{F} N_{f}\right] \frac{C_{F} a^{2}}{6750} \\
+ & {\left[\left(707616000 \zeta_{3}+7527909825\right) C_{A}^{2}-\left(2122848000 \zeta_{3}+1373507730\right) C_{A} C_{F}\right.} \\
& \quad-\left(4626720000 \zeta_{3}+1841332000\right) C_{A} T_{F} N_{f}+\left(1415232000 \zeta_{3}-684744816\right) C_{F}^{2} \\
& \left.+\left(4626720000 \zeta_{3}-3910683210\right) C_{F} T_{F} N_{f}-320975800 T_{F}^{2} N_{f}^{2}\right] \frac{C_{F} a^{3}}{42525000} \\
+ & O\left(a^{4}\right)
\end{aligned}
$$

and

$$
\begin{aligned}
\gamma_{7}^{T}(a)=\frac{258}{35} & C_{F} a+\left[75266555 C_{A}-15484767 C_{F}-33149830 T_{F} N_{f}\right] \frac{C_{F} a^{2}}{2315250} \\
+ & {\left[\left(3517994592000 \zeta_{3}+38365845513450\right) C_{A}^{2}\right.} \\
& -\left(10553983776000 \zeta_{3}+5978407701105\right) C_{A} C_{F} \\
& -\left(24084527040000 \zeta_{3}+9039144860900\right) C_{A} T_{F} N_{f} \\
& +\left(7035989184000 \zeta_{3}-4192441946262\right) C_{F}^{2} \\
& +\left(24084527040000 \zeta_{3}-20698675427220\right) C_{F} T_{F} N_{f} \\
& \left.-1651311191600 T_{F}^{2} N_{f}^{2}\right] \frac{C_{F} a^{3}}{204205050000}+O\left(a^{4}\right) .
\end{aligned}
$$


The complete set of three loop transversity anomalous dimensions in $\overline{\mathrm{MS}}$ and $\mathrm{RI}^{\prime}$ are given in $[18,19,20,24]$.

\section{Conclusions}

We conclude with a few brief remarks. First, the three loop anomalous dimensions are available for the transversity operator for each moment up to $n=8$ in the $\overline{\mathrm{MS}}$ scheme and $n=7$ for the lattice motivated RI' scheme. The former in particular will provide important independent checks for future explicit arbitrary moment evaluations of the three loop anomalous dimensions. A byproduct of the overall project, $[18,19,20]$, has been the provision of the finite parts of a Green's function which are necessary for lattice measurements of matrix elements. The three loop perturbative information is essential to obtaining more precise numerical estimates. In addition we have given the (new) $\mathrm{RI}^{\prime}$ anomalous dimensions for the $n=5$ and 6 Wilson operators at three loops. Whilst it is in principle possible to continue with the computation of the transversity higher moments to $n=9$ and beyond, the present method has become too tedious. This is primarily due to the increase in the number of free Lorentz indices on the operator which was originally required for the lattice comparison. Moreover, the actual computation time as indicated for $n=8$ in the Feynman gauge has already become unacceptably long. An explicit arbitrary $n$ calculation exploiting the algorithm of $[1,2,3,4]$ would achieve all moment information during one run, at possibly a computation time which is not too dissimilar from that for one high moment.

\section{Acknowledgments}

The author thanks Dr P.E.L. Rakow and Dr C. McNeile for valuable discussions.

\section{References}

[1] S. Moch, J.A.M. Vermaseren \& A. Vogt, Nucl. Phys. B688 (2004), 101.

[2] S. Moch, J.A.M. Vermaseren \& A. Vogt, Nucl. Phys. B691 (2004), 129.

[3] S. Moch, J.A.M. Vermaseren \& A. Vogt, Nucl. Phys. Proc. Suppl. 135 (2004), 137.

[4] S. Moch, J.A.M. Vermaseren \& A. Vogt, Phys. Lett. B606 (2005), 123.

[5] J.A.M. Vermaseren, math-ph/0010025.

[6] S.A. Larin, T. van Ritbergen \& J.A.M. Vermaseren, Nucl. Phys. B427 (1994), 41.

[7] S.A. Larin, P. Nogueira, T. van Ritbergen \& J.A.M. Vermaseren, Nucl. Phys. B492 (1997), 338.

[8] S.G. Gorishny, S.A. Larin, L.R. Surguladze \& F.K. Tkachov, Comput. Phys. Commun. 55 (1989), 381.

[9] S.A. Larin, F.V. Tkachov \& J.A.M. Vermaseren, “The Form version of Mincer”, NIKHEF-H-91-18.

[10] A. Rétey \& J.A.M. Vermaseren, Nucl. Phys. B604 (2001), 281.

[11] J. Blümlein \& J.A.M. Vermaseren, Phys. Lett. B606 (2005), 130.

[12] J.P. Ralston \& D.E. Soper, Nucl. Phys. B152 (1979), 109.

[13] R.L. Jaffe \& X. Ji, Phys. Rev. Lett. 67 (1991), 552; Nucl. Phys. B375 (1992), 527. 
[14] J.I. Cortes, B. Pire \& J.P. Ralston, Z. Phys. C55 (1992), 409.

[15] M. Göckeler, R. Horsley, H. Oelrich, H. Perlt, D. Petters, P.E.L. Rakow, A. Schäfer, G. Schierholz \& A. Schiller, Nucl. Phys. B544 (1999), 699.

[16] S. Capitani, M. Göckeler, R. Horsley, H. Perlt, P.E.L. Rakow, G. Schierholz \& A. Schiller, Nucl. Phys. B593 (2001), 183.

[17] M. Göckeler, R. Horsley, D. Pleiter, P.E.L. Rakow, A. Schäfer and G. Schierholz, Nucl. Phys. Proc. Suppl. 119 (2003), 23.

[18] J.A. Gracey, Nucl. Phys. B662 (2003), 247.

[19] J.A. Gracey, Nucl. Phys. B667 (2003), 242.

[20] J.A. Gracey, JHEP 0610 (2006), 040.

[21] G. Martinelli, C. Pittori, C.T. Sachrajda, M. Testa \& A. Vladikas, Nucl. Phys. B445 (1995), 81.

[22] E. Franco \& V. Lubicz, Nucl. Phys. B531 (1998), 641

[23] K.G. Chetyrkin \& A. Rétey, Nucl. Phys. B583 (2000), 3.

[24] J.A. Gracey, Phys. Lett. B643 (2006), 374.

[25] A. Hayashigaki, Y. Kanazawa \& Y. Koike, Phys. Rev. D56 (1997), 7350.

[26] F. Baldracchini, N.S. Craigie, V. Roberto \& M. Socolovsky, Fortschr. Phys. 30 (1981), 505.

[27] X. Artru \& M. Mekhfi, Z. Phys. C45 (1990), 669.

[28] W. Vogelsang, Phys. Rev. D57 (1998), 1886.

[29] J. Blümlein, Eur. Phys. J. C20 (2001), 683.

[30] P. Nogueira, J. Comput. Phys. 105 (1993), 279.

[31] G. 't Hooft, Nucl. Phys. B61 (1973), 455.

[32] W.E. Caswell \& F. Wilczek, Phys. Lett. B49 (1974), 291.

[33] S.A. Larin \& J.A.M. Vermaseren, Phys. Lett. B303 (1993), 334.

[34] J.C. Collins, Renormalization (Cambridge University Press, 1984). 